\title{
Sur le plongement de certaines variétés riemaniennes à deux dimensions dans l'espace euclidien à quatre dimensions (*).
}

\author{
Par $\dagger$ Paul Vincensini (Marseille)
}

\begin{abstract}
Sunto. - Poggiando su una rappresentazione delle trasformazioni puntuali del piano euclideo $E^{\mathrm{B}}$ mediante superficie dello spazio euclideo a 4 dimensioni $E_{4}$, il cui principio è stato esposto nella memoria [1] di questi Annali, si studia un modo particolare d'immersione di aleune varietà riemmaniane a due dimensioni nell' $E_{4}$. Il caso delle realizzazioni reali concrete di tali varietè, sia nell' $E_{4}$ o nella ipersfera unitaria dell' $E_{4}$ (nello spazio ellitico a tre dimensioni) è specialmente esaminato.
\end{abstract}

\section{I. - Introduction.}

Cet article a pour objet l'étude d'un mode particulier de plongement de certaines variétés riemaniennes à deux dimensions dans l'espace euclidien à quatre dimensions. Les variétés envisagées seront regardées comme définies par l'ensemble de deux variables $(u, v)$ et d'une forme différentielle quadratique

$$
d s^{2}=E d u^{2}+2 F d u d v+G d v^{2}, \quad(E, F, G=\text { fonet. de } u \text { et } v) .
$$

Nous dirons qu'une telle variété a été plongée dans un espace euclidien à un nombre quelconque de dimensions, si l'on a pu obtenir explicitement, dans cet espace, une surface à laquelle la métrique euclidienne de l'espace de plongement confère le $d s^{2}$ envisagé. La surface ainsi obtenue fournit alors une représentation concrète (ou une application), dans l'espace euclidien, de la variété donnée.

On connait la difficulté du problème de la détermination des surfaces d'un espace euclidien admettant un $d s^{2}$ donné. Dans l'espace euclidien à trois dimensions ce problème dépend d'une équation de Monge-Ampère, qui n'est complètement intégrable de façon explicite que pour cinq types, d'ailleurs bien connus, de $d s^{2}$. Dans des travaux antérieurs ([2], [3]) nous avons montré comment, la mise d'un $d s^{2}$ sous une forme déterminée (la forme (3) du n. II), permet toujours (aucune distinction entre le réel et l'imaginaire n'étant faite) d'obtenir explicitement une surface (et parfois une infinité de surfaces isométriques entre-elles) représentative du $d s^{2}$ envisagé dans l'espace euclidien $E_{4}$ à quatre dimensions.

(*) Entrata in Redazione il 17 dicembre 1977.

$\dagger$ Deceduto a Marseille il 9 agosto 1978. Bozze rivedute dal Comitato di Redazione. 
La méthode exposée dans les travaux en question fournit, dans certains cas, un moyen de construire dans $E_{4}$ des représentations euclidiennes de variétés riemaniennes à deux dimensions non représentables explicitement dans l'espace euclidien ordinaire à trois dimensions $E_{3}$. En outre, dans le cas où un $d s^{2}$ donné aurait une représentation concrète suivant une surface $\$$ de $E_{3}$, la méthode offre la possibilité d'appliquer isométriquement (et parfois d'une façon continue) cette surface dans $E_{4}$.

Dans les travaux précédents, les variables $u$ et $v$ intervenant dans (1) ont une signification géométrique bien déterminée (qui sera rappelée au $\mathrm{n}$. III), et les $d s^{2}$ envisagés affectent des formes canoniques, dites unifonctionnelles, ne mettant en jeu, dans leurs coefficients, qu'une fonction des deux variables $u, v$ et ses dérivées. Nous nous plaçons ici dans le cas général où, $u$ et $v$ étant quelconques, le $d s^{2}$ de la variété envisagée a la forme générale (1), pour en déduire de nouveaux cas d'application de variétés riemaniennes bidimentionnelles (et notamment de surfaces de $E_{3}$ ) dans $E_{4}$.

\section{II. - Les formules fondamentales. Rappel de résultats.}

L'espace euclidien ordinaire $E_{3}$ est supposé être l'hyperplan $x_{4}=0$ de l'espace euclidien $E_{4}$ rapporté au repère orthonormé $\left(O x_{1} x_{2} x_{3} x_{4}\right)$.

Considérons l'ensemble des $\infty^{4}$ droites de $E_{3}$. L'une quelconque, $D$, de ces droites coupe respectivement les plans $x_{3}=0, x_{3}=1$ de $E_{3}$ en $A$ et $B$. Soit $A^{\prime}$ la projection orthogonale de $B$ sur le plan $E_{2}\left(O x_{1} x_{2}\right)$. L'ensemble des couples ordonnés $(A$, $A^{\prime}$ ) de points du plan $E_{2}$ et celui des droites $D$ de $E_{\mathrm{a}}$ non parallèles à $E_{2}$ se correspondent biunivoquement, et si $\left(\xi_{1}, \xi_{2}, 0\right),\left(\eta_{1}, \eta_{2}, 1\right)$ sont respectivement les coordonnées des points $A$ et $B$ de $E_{3}$, et par suite $\left(\xi_{1}, \xi_{2}\right),\left(\eta_{1}, \eta_{2}\right)$ celles de $A$ et $A^{\prime}$ dans $E_{2}$ $\left(O x_{1} x_{2}\right)$, les équations

$$
\left\{\begin{array}{l}
x_{1}=\left(\xi_{1}+\xi_{2}-\eta_{1}\right) / 2, \\
x_{2}=\left(\xi_{1}-\xi_{2}-\eta_{1}\right) / 2 i, \\
x_{3}=\left(\xi_{2}-\xi_{1}-\eta_{2}\right) / 2, \\
x_{4}=\left(\xi_{2}+\xi_{1}-\eta_{2}\right) / 2 i,
\end{array}\right.
$$

définissent une application biunivoque de l'ensemble des comples ordonnés de points de $E_{2}$ (ou des droites $D$ de $E_{3}$ ) dans $E_{1}$, qui est précisément celle qui nous a servi dans les travaux cités et que nous adopterons dans toute la suite.

Eu égard aux applications relatives au plongement de variétés à deux dimensions dans $E_{4}$ les deux interprétations géométriques suivantes des formules (2) jouent un rôle important:

Si l'on regarde $\xi_{1}, \xi_{2}, \eta_{1}, \eta_{2}$ comme des fonctions de deux variables indépendantes $u$ et $v$ (lesquelles, par exemple, peuvent être $\xi_{1}$ et $\xi_{2}$ ), on obtient une transfor- 
mation ponctuelle $\mathcal{C}\left[A\left(\xi_{1}, \xi_{2}\right) \rightarrow A^{\prime}\left(\eta_{1}, \eta_{2}\right)\right]$ dans $E_{2}$. Le point $M\left(x_{1}, x_{2}, x_{3}, x_{4}\right)$ de $E_{4}$ défini par les formules (2) décrit alors une surface $\$$, et l'on peut dire que ces formules appliquent (biunivoquement) l'ensemble des transformations ponetuelles de $E_{2}$ sur l'ensemble des surfaces $S$ de $E_{4}$.

Si $\left(\xi_{1}, \xi_{2}\right),\left(\eta_{1}, \eta_{2}\right)$ sont regardées, ainsi qu'il a été expliqué plus haut, comme définissant une droite $D$ de $E_{3}$, cette droite, dépendant des deux paramètres $u$ et $v$, engendre une congruence de $E_{3}$, et les formules (2) définissent alors une application des congruences rectilignes de $E_{3}$ sur les surfaces de $E_{4}$. Et cette application peut être étendue aux complexes de $E_{3}$ ou à l'espace règlé $E_{3}$ tout entier si l'on suppose $\xi_{1}, \xi_{2}, \eta_{1}, \eta_{2}$ fonctions de 3 ou 4 paramètres arbitraires.

On vérifie aussitôt au moyen des formules (2) que dans l'application de l'espace $E_{3}$ règlé sur $E_{4}[1]$, à deux droites concourantes de $E_{3}$ correspondent biunivoquement deux points de $E_{4}$ situés sur une même droite isotrope, les développables de $E_{3}$ et les courbes isotropes de $E_{4}$ se correspondant ainsi biunivoquement.

Quant au point de vue consistant à regarder les formules (2) comme définissant une application des transformations ponetuelles $A\left(\xi_{1}, \xi_{2}\right) \rightarrow A^{\prime}\left(\eta_{1}, \eta_{2}\right)$ de $E_{2}$ sur des surfaces, il conduit simplement à une forme canonique intéressante $d u d \delta^{2}$ d'une variété riemanienne quelconque à deux dimensions. Le calcul de la distance, dans $E_{4}$, des images $M$ et $M+d M$ des deux points infiniment voisins $A\left(\xi_{1}, \xi_{2}\right), A^{\prime}\left(\eta_{1}, \eta_{2}\right)$ et $\overline{A+d A}\left(\xi_{1}+d \xi_{1}, \xi_{2}+d \xi_{2}\right), \overline{A^{\prime}+d A^{\prime}}\left(\eta_{1}+d \eta_{1}, \eta_{2}+d \eta_{2}\right)$, fait au moyen des formules (2), donne

$$
d s^{2}=d M^{2}=d \xi_{1} d \eta_{2}-d \xi_{2} d \eta_{1}
$$

ce qui montre que $d s^{2} n^{\prime}$ est autre chose que le produit extérieur des deux vecteurs infinitésimaux $\boldsymbol{d A}, \boldsymbol{d \boldsymbol { A } ^ { \prime }}$.

Lorsque $\xi_{1}, \ldots, \eta_{2}$ sont des fonctions quelconques de deux paramètres $u$ et $v$, et que par suite $\mathcal{G}\left(A \rightarrow A^{\prime}\right)$ est la transformation ponctuelle la plus générale du plan $E_{2}$, l'expression (3) du $d s^{2}$ fournit la forme canonique annoncée.

Notons aussi l'interprétation suivante du carré $O \bar{M}^{2}$ de la distance à l'origine, dans $E_{4}$, du point $M$ image du couple $\left(A, A^{\prime}\right)$ de $E_{2}$.

Les (2) montrent que l'on a

$$
o \bar{M}^{2}=\xi_{1} \eta_{2}-\xi_{2} \eta_{1}
$$

et que par suite $O \bar{M}^{2}$ représente le produit extérieur des deux vecteurs $O \boldsymbol{A}, O \boldsymbol{A}^{\prime}$, c'est-à-dire l'aire du parallèlogramme construit dans le plan $E_{2}$ sur $O A$ et $O A^{\prime}$. D'où il résulte, en particulier, que toutes les transformations $\mathfrak{C}\left(A \rightarrow A^{\prime}\right)$ du plan $E_{2}$ pour lesquelles le triangle $\left(O A A^{\prime}\right)$ conserve une aire constante, ont pour images des surfaces situées sur une hypersphère de $E_{4}$ centrée en $O$, remarque qui se révèle utile dans le problème de la recherche de variétés riemaniennes (de surfaces de $E_{3}$ notamment) susceptibles d'un plongement isométrique dans une hypersphère de $E_{4}$ auquel il a été precédemment fait allusion. 


\section{III. - Formules générales de plongement.}

On obtient ces formules en exprimant l'équivalence de la forme différentielle quadratique (1) qui définit l'élément linéaire de la variété envisagée, et de la forme (3) où les quatre quantités $\left(\xi_{1}, \ldots, \eta_{2}\right)$ sont des fonctions inconnues des deux variables $u$ et $v$ figurant dans (1). Cette équivalence se traduit, comme on le voit aussitôt, par le système

$$
\left\{\begin{array}{l}
\frac{\partial \xi_{1}}{\partial u} \frac{\partial \eta_{2}}{\partial u}-\frac{\partial \xi_{2}}{\partial u} \frac{\partial \eta_{1}}{\partial u}=E \\
\frac{\partial \xi_{1}}{\partial v} \frac{\partial \eta_{2}}{\partial v}-\frac{\partial \xi_{2}}{\partial v} \frac{\partial \eta_{1}}{\partial v}=G \\
\frac{\partial \xi_{1}}{\partial u} \frac{\partial \eta_{2}}{\partial v}+\frac{\partial \xi_{1}}{\partial v} \frac{\partial \eta_{2}}{\partial u}-\frac{\partial \xi_{2}}{\partial u} \frac{\partial \eta_{1}}{\partial v}-\frac{\partial \xi_{2}}{\partial v} \frac{\partial \eta_{1}}{\partial u}=2 F
\end{array}\right.
$$

L'intégrabilité théorique du système (4) est assurée, ne serait-ce qu'en raison de la possibilité bien connue de plongement de la variété générale à $n$ dimensions dans un espace euclidien à $n(n+1) / 2$ dimensions an moins.

$\mathrm{Si}\left[x_{1}(u, v), \ldots, x_{4}(u, v)\right]$ définissent, dans $E_{4}$, une surface ayant un $d s^{2}$ donné de la forme générale (1), les équations (2) font connaître les quatre fonctions $\left(\xi_{1}, \ldots, \eta_{2}\right)$ des deux variables $u$ et $v$ vérifiant le système différentiel (4). Mais la possibilité d'obtenir explicitement des solutions $\left(\xi_{1}, \xi_{2}, \eta_{1}, \eta_{2}\right)$ du système (4) est liée à la forme analytique des fonctions $E, F, G$ des variables $u$ et $v$ figurant dans (1), et nous nous proposons, dans ce qui suit, d'examiner un certain nombre de cas, suggérés par l'origine géométrique des quantités $\left(\xi_{1}, \ldots, \eta_{2}\right)$ définies au n. II, où cette possibilité a lieu.

Supposons, comme dans les travaux cités au n. II, que les paramètres $(u, v)$ qui interviennent dans la transformation ponctuelle $\mathscr{C}\left(A \rightarrow A^{\prime}\right)$ du plan $E_{2}$ soient précisément les coordonnées $\left[\xi_{1}=u, \xi_{2}=v\right]$ du point générique $A$ du plan $E_{2}$. Le système (4) associé au plongement $d u d s^{2}(3)$ dans $E_{4}$ définit alors les deux fonctions inconnues $\eta_{1}(u, v), \eta_{2}(u, v)$ au moyen du nouveau système

$$
\left\{\begin{array}{l}
\frac{\partial \eta_{2}}{\partial u}=E \\
\frac{\partial \eta_{1}}{\partial v}=-G \\
\frac{\partial \eta_{2}}{\partial v}-\frac{\partial \eta_{1}}{\partial u}=2 F
\end{array}\right.
$$

Un tel système n'est généralement pas intégrable. Son intégration exige une cer- 
taine relation entre les coefficients $E, F, G$, que l'on obtient de la façon suivante.

Des deux premières équations (5) on déduit

$$
\left\{\begin{array}{l}
\eta_{1}=-\int_{v_{0}}^{v} G d v+\lambda(u) \\
\eta_{2}=\int_{u_{0}}^{u} E d u+\mu(v)
\end{array}\right.
$$

la 3-ème équation (5) donne alors

$$
\int_{u_{0}}^{u b} \frac{\partial E}{\partial v} d u+\int_{v_{a}}^{v} \frac{\partial G}{\partial u} d v-2 F=\lambda^{\prime}(u)-\mu^{\prime}(v),
$$

et l'on doit done avoir

$$
\frac{\partial^{2}}{\partial u \partial v}\left[\int_{v_{0}}^{v} \frac{\partial E}{\partial v} d u+\int_{u_{0}}^{u} \frac{\partial G}{\partial u} d v-2 F\right]=0
$$

soit:

$$
\frac{\partial^{2} E}{\partial v^{2}}+\frac{\partial^{2} G}{\partial u^{2}}-2 \frac{\partial^{2} F}{\partial u \partial v}=0
$$

Réciproquement d'ailleurs, si (8) est vérifiée, le 1-er membre de (7) est de la forme $\lambda^{\prime}(u)-\mu^{\prime}(v), \lambda^{\prime}$ et $\mu^{\prime}$ étant des fonctions déterminées de $u$ et $v$ respectivement, lesquelles définissent $\lambda$ et $\mu$ à des constantes additives près, qui, intervenant additivement dans les expressions (2) de $\left(x_{1}, x_{2}, x_{3}, x_{4}\right)$ comme le montrent les expressions (6) de $\eta_{1}, \eta_{2}$, n'introduisent qu'une translation dans $\pi_{4}$ et peuvent par suite être négligées.

Les équations (6) où $\lambda$ et $\mu$ ont les valeurs trouvées définissent alors une transformation ponctuelle

$$
\mathcal{G}\left\{\begin{array}{l|l}
\xi_{1}=u \\
\xi_{2}=v
\end{array} \rightarrow A^{\prime} \mid \begin{array}{l}
\eta_{1}=-\int_{v_{0}}^{v} G d v+\lambda(u) \\
\eta_{2}=\int_{u_{0}}^{u} E d u+\mu(v)
\end{array}\right\}
$$

du plan $E_{2}$, dont la surface image dans $E_{4}$, définie par les équations (2), admettra le $d s^{2}(1)$ donné. 
Les équations de cette surface sont:

(9)

$$
\left\{\begin{array}{l}
x_{1}=\left[u+v+\int_{v_{0}}^{v} G d v-\lambda(u)\right] / 2 \\
x_{2}=\left[u-v+\int_{v_{0}}^{v} G d v-\lambda(u)\right] / 2 i \\
x_{3}=\left[v-u-\int_{u_{0}}^{u} E d u-\mu(v)\right] / 2 \\
x_{4}=\left[v+u-\int_{u_{0}}^{u} E d u-\mu(v)\right] / 2 i
\end{array}\right.
$$

On peut done énoncer le résultat suivant:

Si les coefficients $E, F, G d u d s^{2}(1)$ vérifient la relation

$$
\frac{\partial^{2} E}{\partial v^{2}}+\frac{\partial^{2} G}{\partial u^{2}}-\frac{\partial^{2} F}{\partial u \partial v}=0
$$

les variétés supports de ce $d s^{2}$ admettent un plongement explicite dans $E_{4}$, défini par les équations (9), où les fonctions $\lambda(u)$ et $\mu(v)$ sont uniquement déterminées (à des constantes négligeables près) par le développement du premier membre de l'équation (7).

Parmi les $d s^{2}$ vérifiant (8) figurent les deux suivants

$$
\begin{cases}d s^{2}=E\left(d u^{2}+d v^{2}\right), & E=\text { fonction harmonique de } u \text { et } v \\ d s^{2}=2 F d u d v, & F=U(u)+V(v)\end{cases}
$$

dont le plongement dans $E_{4}$ a fait l'objet d'une étude publiée dans la revue de l'Université d'Istanbul (vol. 42, 1977).

Un autre type de $d s^{2}$ vérifiant (8), et dont ou peut par suite réaliser le plongement euclidien dans $E_{4}$ au moyen des formules générales (9) est le suivant:

$$
d s^{2}=\left[a v^{2}+V_{1} v+U_{2}\right] d u^{2}+\left[-a u^{2}+V_{1} u+V_{2}\right] d v^{2},
$$

où $a$ est une constante arbitraire, et où $\left(U_{1}, U_{2}\right),\left(V_{1}, V_{2}\right)$ sont des couples de foncm tions arbitraires de $u$ et $v$ respectivement.

Ici:

$$
E=a v^{2}+V_{1} v+U_{2}, \quad G=-a u^{2}+V_{1} u+V_{2}, \quad F=0
$$


PaUl Vincensini: Sur le plongement de certaines variétés riemaniennes, etc.

97

on a done

$$
\begin{aligned}
& \int_{u_{0}}^{u} \frac{\partial E}{\partial v} d u=2 a u v-2 a u_{0} v+\int_{u_{0}}^{u} U_{1} d u \\
& \int_{v_{0}}^{v} \frac{\partial G}{\partial u} d v=-2 a u v+2 a v_{0} u+\int_{v_{0}}^{v} V_{1} d v
\end{aligned}
$$

et par suite

$$
\int_{u_{0}}^{u} \frac{\partial E}{\partial v} d u+\int_{v_{0}}^{v} \frac{\partial G}{\partial u} d v=\left(2 a v_{0} u+\int_{u_{0}}^{u} \nabla_{1} d u\right)-\left(2 a u_{0} v-\int_{v_{0}}^{v} \nabla_{1} d v\right) .
$$

La relation (7) est bien vérifiée, $\lambda^{\prime}(u)$ et $\mu^{\prime}(v)$ ayant les expressions ci-dessus entre parenthéses, d'où l'on déduit

$$
\begin{aligned}
& \lambda(u)=a v_{0} u^{2}+\iint_{\psi_{0}}^{u} U_{1} d u, \\
& \mu(v)=a u_{0} v^{2}-\iint_{v_{0}}^{v} V_{1} d v .
\end{aligned}
$$

Les expressions $(6)$ de $\eta_{1}, \eta_{2}$ donnent alors pour $\eta_{1}, \eta_{2}$ :

$$
\begin{aligned}
& \eta_{1}=a u^{2} v-u \int_{v_{0}}^{v} V_{1} d v-\int_{v_{0}}^{v} V_{2} d v+\iint_{v_{v_{0}}}^{u} U_{1} d u, \\
& \eta_{2}=a v^{2} u+v \int_{u_{0}}^{u} U_{1} d u+\int_{u_{0}}^{u} U_{2} d u-\iint_{v_{0}}^{v} V_{1} d v,
\end{aligned}
$$

et les équations (2) définissant les surfaces de $E_{4}$ admettant le $d s^{2}$ (11) s'écrivent:

$$
\left\{\begin{array}{l}
x_{1}=\left[u+v-a u^{2} v+u \int_{v_{0}}^{v} V_{1} d v+\int_{v_{0}}^{v} V_{2} d v-\iint_{u_{0}}^{u} U_{1} d u\right] / 2, \\
x_{2}=\left[u-v-a u^{2} v+u \int_{v_{0}}^{v} V_{1} d v+\int_{v_{0}}^{v} V_{2} d v-\iint_{u_{0}}^{u} U_{1} d u\right] / 2 i, \\
x_{3}=\left[v-u-a v^{2} u-v \int_{u_{0}}^{u} U_{1} d u-\int_{u_{0}}^{u} U_{2} d u+\iint_{v_{0}}^{v} V_{1} d v\right] / 2, \\
x_{4}=\left[v+u-a v^{2} u-v \int_{u_{0}}^{u} U_{1} d u-\int_{u_{0}}^{u t} U_{2} d u+\iint_{v_{0}}^{v} V_{1} d v\right] / 2 i .
\end{array}\right.
$$


Si on laisse de coté dans les expressions de $x_{1}, \ldots, x_{4}$ les constantes additives qui, comme on l'a dit, correspondent à des translations de la surface (12) dans $E_{4}$, on constate que, pour un $d s^{2}$ donné de la forme (11), les (12) représentent une famille de surfaces isométriques dépendant de deux paramètres, lesquels sont introduits par les quadratures $\int_{u_{0}}^{u} U_{1} d u$ et $\int_{v_{0}}^{v} V_{1} d V$.

Si $a=U_{1}=V_{1}=0$, les équations (12) qui, en posant $U=\int_{u_{0}}^{u_{u}} U_{2} d u, \mathcal{V}=\int_{v_{0}}^{v} V_{2} d v$ s'écrivent

$$
\left\{\begin{array}{l}
x_{1}=(u+v+\cup) / 2, \\
x_{2}=(u-v+\cup) / 2 i, \\
x_{3}=(v-u-\cup) / 2, \\
x_{4}=(v+u-\cup) / 2 i,
\end{array}\right.
$$

définissent une application isométrique $d u$ plan euclidien $E_{2}$ dans $E_{4}$.

Les surfaces (13) sont des surfaces de translation, et parmi elles figurent des quadriques de $E_{4}$. La possibilité d'appliquer isométriquement le plan euclidien sur des quadriques de $E_{4}$ a été mise en evidence pour la 1-ère fois par C. GUTCHARD [4]. On voit que cette propriété s'étend, entre autres, à toute une infinité de surfaces algébriques, obtenues en prenant pour $U_{2}$ et $V_{2}$ des polynômes quelconques en $u$ et $v$ respectivement.

Si $a=U_{1}=0$, si l'on a en outre $U_{2}=1, \nabla_{1}=2$, et si l'on pose $V_{2}=2$, le $d s^{2}$ (11) se présente sous la forme

$$
d s^{2}=d u^{2}+2(u+\cup) d v^{2},
$$

qui a fait l'objet d'études approfondies de la part de J. Weingarten [5], G. DARBouX [6], E. Goursat [7], E. Baroni [8], et qui, pour des particularisations bien connues de la fonction $V$ donne les cinq types de $d s^{2}$ dont toutes les surfaces représentatives dans $E_{3}$ peuvent être explicitement déterminées. Dans les travaux des auteurs cités les $d s^{2}$ de la forme (14) apparaissent à travers une étude approfondie de l'équation de Monge-Ampère dont dépend le problème général de la déformation des surfaces [9]. Nous les avons retrouvés à notre tour d'une manière nouvelle [10] à propos d'un problème concernant la déformation de certaines variétes règlées. L'étude actuelle ajoute encore à leur intéret, en montrant notamment comment on peut obtenir explicitement, dans $E_{4}$, sans intégration d'aucune équation différentielle, pour chaque $d s^{2}$ de la forme générale (11), une famille de $\infty^{1}$ surfaces représentatives dans $E_{4}$.

Si $v$ est une constante, que moyennant un changement de variable sur $u$ on peut supposer nulle, on a $10 d s^{2}$

$$
d s^{2}=d u^{2}+2 u d v^{2}
$$


qui convient aux surfaces applicables sur les développées des surfaces minima de $E_{3}$; les formules (12) qui appliquent isométriquement ces surfaces dans $E_{4}$ s'écrivent

$$
\left\{\begin{array}{l}
x_{1}=[C u+v+2 u v] / 2, \\
x_{2}=[C u-v+2 u v] / 2 i, \\
x_{3}=\left[C v-2 u+v^{2}\right] / 2, \\
x_{4}=\left[C v+v^{2}\right] / 2 i,
\end{array}\right.
$$

et l'on retrouve un résultat déja signalé dans une communication faite au Congrès International de Nice suivant lequel:

Les développées des surfaces minima de $E_{3}$ sont isométriquement applicables sur des quadriques de $E_{4}$.

Notons aussi la possibilité de plongement, au moyen des formules générales (12), $d u d s^{2}$ de Liouville

$$
d s^{2}=\left(u^{2}-v^{2}\right)\left(d u^{2}+d v^{2}\right),
$$

obtenu en faisant: $a=-1, U_{1}=V_{1}=0, U_{2}=u^{2}, V_{2}=-v^{2}$.

Les équations (12) deviennent dans ce dernier cas

$$
\left\{\begin{array}{l}
x_{1}=\left[u+v+u^{2} v-v^{3} / 3\right] / 2, \\
x_{2}=\left[u-v+u^{2} v-v^{3} / 3\right] / 2 i, \\
x_{3}=\left[v-u+v^{2} u-u^{3} / 3\right] / 2, \\
x_{4}=\left[v+u+v^{2} u-u^{3} / 3\right] / 2 i,
\end{array}\right.
$$

et représentent une surface cubique de l'espace à 4 dimensions.

\section{IV. - Les $d s^{2}$ isothermiques harmoniques et leur plongement suivant des surfaces minimales du type de Riemann de $E_{4}$.}

Si l'on ne suppose pas que les variables $u, v$ sont les coordonnées dans $E_{2}$ du 1-er point $A$ du couple $\left(A, A^{\prime}\right)$ d'image $M\left(x_{1}, x_{2}, x_{3}, x_{4}\right)$ dans $E_{4}$, le problème du plongement $d u d s^{2}$ (1) dans $E_{4}$ dépend du système général (4) du n. III. Ce système définit, au moyen des deux paramètres $u$ et $v$, les différents couples de points de $E_{2}$ se correspondant dans la transformation $\mathfrak{C}\left[A\left(\xi_{1}, \xi_{2}\right) \rightarrow A^{\prime}\left(\eta_{1}, \eta_{2}\right)\right]$ dont l'image dans $E_{1}$ représente le $d s^{2}$ donné. Son intégration introduira en général une fonetion arbitraire des variables $(u, v)$, et le plongement sera défini à ce degré d'arbitraire près.

L'intégration du système (4) est en général difficile. Aussi, plutôt que d'envisager le problème d'immersion qui nous occupe sous la forme générale précédente, 
porterons nous notre attention sur la recherche des $d s^{2}$ dont le plongement dans $E_{4}$ se trouve facilité par quelque particularité géométrique opportune de la transformation ponctuelle $\mathscr{C}\left(A \rightarrow A^{\prime}\right)$ correspondante.

Supposons par exemple que $\mathfrak{G}$ soit une transormation conforme directe réelle de $E_{2}, \xi_{1}$ et $\xi_{2}$ étant les coordonnées $\left(\xi_{1}=u, \xi_{2}=v\right)$ du point $A$. On aura donc

$$
\left\{\begin{array} { l l } 
{ \frac { \partial \xi _ { 1 } } { \partial u } = 1 , } & { \frac { \partial \xi _ { 1 } } { \partial v } = 0 , } \\
{ \frac { \partial \xi _ { 2 } } { \partial u } = 0 , } & { \frac { \partial \xi _ { 2 } } { \partial v } = 1 , }
\end{array} \left\{\begin{array}{l}
\frac{\partial \eta_{1}}{\partial u}=\frac{\partial \eta_{2}}{\partial v}, \\
\frac{\partial \eta_{1}}{\partial v}=-\frac{\partial \eta_{2}}{\partial u} ;
\end{array}\right.\right.
$$

les deux premières équations (4) montrent que

$$
\frac{\partial \eta_{2}}{\partial u}=E, \quad \frac{\partial \eta_{1}}{\partial v}=-G
$$

d'où, d'après (15) $E=G$, et la 3 -ème (4) donne

$$
\frac{\partial \eta_{1}}{\partial u}-\frac{\partial \eta_{2}}{\partial v}=2 F=0
$$

Il s'agit donc du plongement dans $E_{\mathrm{a}}$ de $d s^{2}$ isothermiques

$$
d s^{2}=E\left(d u^{2}+d v^{2}\right)
$$

Si l'on pose

$$
\frac{\partial \eta_{\mathrm{x}}}{\partial u}=\frac{\partial \eta_{2}}{\partial v}=\theta(u, v)
$$

les expressions des dérivées des fonctions harmoniques conjuguées $\eta_{1}, \eta_{2}$ prennent les formes

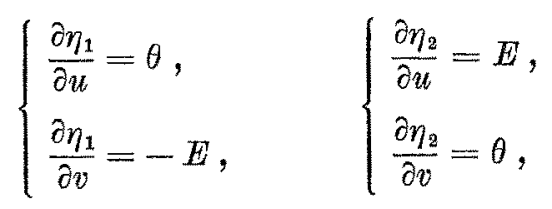

et les conditions d'intégrabilité en $\eta_{1}$ et $\eta_{2}$ exigent comme l'on voit que $\theta+i E$ soit une fonction analytique de $u+i v$, et que par suite $E$ soit une fonction harmonique de $u, v,-\theta$ étant une fonction harmonique conjuguée de $E$.

S'il en est ainsi les équations (17) donnent pour $\eta_{1}$ et $\eta_{2}$

$$
\eta_{1}=\int \theta d u-E d v, \quad \eta_{2}=\int E d u+A d v .
$$


Pour que le plongement $d u d s^{2}$ isothermique (16) au moyen des formules (2) $d u$ n. II soit possible, il faut donc, et il suffit, que $E$ soit une fonction harmonique quelconque des deux variables $u$ et $v$. S'il en est ainsi on obtient les formules de plongement en remplaģant, dans les formules (2), $\xi_{1}$ et $\xi_{2}$ par $u$ et $v$ respectivement et $\eta_{1}, \eta_{2}$ par les expressions (18) où $-\theta$ est une fonction harmonique conjuguée de $E$. On trouvera ainsi pour les surfaces de plongement les équations

$$
\left\{\begin{array}{l}
x_{1}=\left[u+v-\int \theta d u-E d v\right] / 2 \\
x_{2}=\left[u-v-\int \theta d u-E d v\right] / 2 i \\
x_{3}=\left[v-u-\int E d u+\theta d v\right] / 2 \\
x_{4}=\left[v+u-\int E d u+\theta d v\right] / 2 i
\end{array}\right.
$$

La fonction $\theta$, pour $E$ fonction harmonique donnée, n'est définie qu'à tue constante additive près, mais il est facile de voir que la variation de cette constante n'introduit qu'un simple déplacement euclidien de la surface (19) dans $E_{4}$.

Si l'on fait le changement de variables $(u=(\alpha+\beta) / 2, v=(\alpha-\beta) / 2 i)$ on constate sans peine que les intégrales figurant dans les équations (19) sont des sommes d'une fonction de $\alpha$ et d'une fonction de $\beta$, et que par suite les surfaces de plongement sont des surfaces de transtation, d'ailleurs à réseaux de translation formés de courbes de longueur nulle planes et situées dans des plans totalement isotropes.

Ces propriétés identifient les surfaces de plongement des $d s^{2}$ isothermiques (16) à coefficient harmonique avec une famille de surfaces minimates de $E_{4}$, que nous avons etudiées antérieurement [2], [11] sous le nom de surfaces minimales du type de Riemann. Ces surfaces jouissent d'un certain nombre de propriétés intéressantes. Contrairement à ce qui a lieu pour les surfaces minimales générales de l'espace euclidien à trois dimensions, il est impossible de déformer isométriquement ces surfaces à l'intérieur de la famille générale des surfaces minimales de $E_{4}$. Ce qui a été dit sur la rigidité de la surface définie par les équations (19) au regard de la variation de la constante arbitraire dont ces équations dépendent, s'inscrit dans le résultat général précédent.

\section{V. - Plongements hypersphériques.}

On peut imposer à la transformation ponctuelle $\mathcal{G}\left(A \rightarrow A^{\prime}\right)$ de $E_{2}$ bien d'autres propriétés que la conformité directe dont il a été question an $\mathrm{n}$. précédent. Nous nous bornerons ici à la considération de l'une d'entre-elles, suggérée par les remarques géométriques exposées au n. II.

On a vu que pour une transformation $\mathscr{G}\left(A \rightarrow A^{\prime}\right)$ donnée dans $E_{2}$, l'aire du parallélogramme construit sur les deux vecteurs $O A, O A^{\prime}$ représente le carré $\left(O M^{2}\right)$ de la distance de l'origine $O$ au point $M$ de $E_{4}$ image du couple ordonné $\left(A, A^{\prime}\right)$. Les 
transformations $\mathscr{G}\left(A \rightarrow A^{\prime}\right)$ pour lesquelles l'aire du triangle $\left(O A A^{\prime}\right)$ conserve une valeur constante ont done pour images des surfaces situées sur une hypersphère de centre $O$ de $E_{4}$, et l'on obtiendra des $d s^{2}$ susceptibles de plongements hypersphériques dans $E_{4}$ au moyen des formules (2) en imposant à $\mathcal{C}$ la condition précédente.

Un cas évident d'invariance de l'aire $\left(O A A^{\prime}\right)$ est celui où les points $A, A^{\prime}$, supposés réels, ont pour coordonnées

$$
A\left\{\begin{array} { l } 
{ \xi _ { 1 } = u , } \\
{ \xi _ { 2 } = v , }
\end{array} \quad A ^ { \prime } \left\{\begin{array}{l}
\eta_{1}=u \\
\eta_{2}=v+1 / u
\end{array}\right.\right.
$$

On a alors, dans $E_{4}, O M^{2}=1$, et l'image de la transformation $\mathcal{G}\left(A \rightarrow A^{\prime}\right)$ est sur l'hypersphère de rayon 1.

Dans le cas actuel on a

$$
d s^{2}=d \xi_{1} d \eta_{2}-d \xi_{2} d \eta_{1}=-\frac{1}{u^{2}} d u^{2}
$$

c'est là le $d s^{2} d^{\prime} u n$ plan isotrope de $E_{3}$, et l'on retrouve le résultat connu suivant lequel les plans isotropes de $E_{3}$, qui sont indéformables dans $E_{3}$, peuvent être appliqués isometriquement sur des surfaces hypersphériques de $\boldsymbol{E}_{4}$.

Les équations générales (2) définissant le plongement du $d s^{2}$ ci-dessus dans l'hypersphère de rayon 1 s'écrivent ici

$$
x_{1}=v / 2, \quad x_{2}=-v / 2 i, \quad x_{3}=-\left(u+\frac{1}{u}\right) / 2, \quad x_{4}=\left(u-\frac{1}{u}\right) / 2 i .
$$

Elles représentent une 2-sphère équatoriale de l'hypersphère de rayon 1, obtenue en coupant celle-ci par l'hyperplan

$$
x_{1}+i x_{2}=0
$$

Si l'on définit les $\infty^{2}$ triangles $\left(O A A^{\prime}\right)$ par

$$
\begin{gathered}
\overline{O A}=u, \quad \widehat{O x_{1}, O A}=v, \quad \text { (coordonnées polaires de } A \text { ) } \\
\widehat{O A, O A^{\prime}}=\theta(u, v), \quad \overline{O A^{\prime}}=f(u, v),
\end{gathered}
$$

leur équivalence (aire commune $=\frac{1}{2}$ ) s'exprime par $u f \sin \theta=1$, ce qui donne pour les coordonnées des points $A$ et $A^{\prime}$

$$
\left\{\begin{array} { l } 
{ \xi _ { 1 } = u \operatorname { c o s } v , } \\
{ \xi _ { 2 } = u \operatorname { s i n } v , }
\end{array} \quad \left\{\begin{array}{l}
\eta_{1}=\cos (\theta+v) / u \sin \theta \\
\eta_{2}=\sin (\theta+v) / u \cos \theta
\end{array}\right.\right.
$$


et pour le $d s^{2}$ de l'image hypersphérique de la transformation $\mathscr{C}\left(A \rightarrow A^{\prime}\right)$ du plan $E_{2}$ :

$$
d s^{2}=d \xi_{1} d \eta_{2}-d \xi_{2} d \eta_{1}=-\frac{1}{u^{2}} d u^{2}-u^{2} \frac{\partial}{\partial u}\left(\frac{\operatorname{Cotg} \theta}{u^{2}}\right)+\left(1-\frac{\partial \operatorname{Cotg} \theta}{\partial v}\right) d v^{2} .
$$

Si l'on définit $\theta$ par la relation $\operatorname{Cotg} \theta=\vee u^{2}$, où $V$ est fonction de $v$ seulement, le $d s^{2}$ précedent affecte la forme orthogonale

$$
d s^{2}=-\frac{1}{u^{2}} d u^{2}+\left(1-V^{\prime} u^{2}\right) d v^{2}
$$

laquelle, restant inaltérée si l'on remplace $u$ par $k u$ et $V$ par $V / k^{2}$, convient à une famille de surfaces isométriques à un paramètre $(k)$ de l'hypersphère de rayon 1 de $E_{4}$, definie par les formules (2) du $n$. II qui, compte tenu des équations (20) s'écrivent

$$
\left\{\begin{array}{l}
x_{1}=\left[\left(\frac{k^{2}-V}{k}\right) u \cos v+\frac{k^{2} u^{2}+1}{k u} \sin v\right] / 2 \\
x_{2}=\left[\left(\frac{k^{2}-V}{k}\right) u \cos v+\left(\frac{-k^{2} u^{2}+1}{k u}\right) \sin v\right] / 2 i \\
x_{3}=\left[-\left(\frac{k^{2} u^{2}+1}{k u}\right) \cos v+\left(\frac{k^{2}-V}{k}\right) u \sin v\right] / 2 \\
x_{4}=\left[\left(\frac{k^{2} u^{2}-1}{k u}\right) \cos v+\left(\frac{k^{2}-V}{k}\right) u \sin v\right] / 2 i
\end{array}\right.
$$

Pour chaque choix de la fonction $V(v)$ les équations (21) définissent une famille de surfaces isométriques sur l'hypersphère de rayon 1 de $E_{4}$.

\section{VI. - Plongements réels. Cas de l'espace elliptique.}

Les plongements de variétés à deux dimensions dans $E_{4}$ obtenus par le procèdé exposé dans ce qui précède sont en général imaginaires. Les surfaces de $E_{4}$ sur lesquelles les variétés en question viennent s'appliquer isométriquement, définies par les formules générales (2), sont en effet imaginaires, et cela que la transformation ponetuellé $\left(\xi_{1}, \xi_{2}\right) \rightarrow\left(\eta_{1}, \eta_{2}\right)$ du plan $E_{2}$ relative à ces plongements soit elle-même réelle ou imaginaire. Demandons nous comment on peut obtenir les variétés donnant lieu à des plongements réels. Ecrivons à cet effet que l'on a

$$
\left\{\begin{array}{l}
\xi_{1}+\xi_{2}-\eta_{1}=2 R, \\
\xi_{1}-\xi_{2}-\eta_{1}=2 i S, \\
\xi_{2}-\xi_{1}-\eta_{2}=2 T, \\
\xi_{2}+\xi_{1}-\eta_{2}=2 i U,
\end{array}\right.
$$


$R, S, T, U$ étant des quantités réelles, fonctions des variables réelles $u, v$, dont la somme des carrés représente, notons le, le carré de la distance au point $O$ du point courant $M$ de la surface (2) de $E_{4}$ sur laquelle a lieu le plongement de la variété envisagée:

$$
\overline{O M}^{2}=R^{2}+S^{2}+T^{2}+U^{2}
$$

Les (22) donnent

$$
\left\{\begin{array}{l}
\xi_{1}=-T+i U, \\
\xi_{2}=R-i S, \\
\eta_{1}=-(T+R)+i(U-S), \\
\eta_{2}=(R-T)-i(U+S) ;
\end{array}\right.
$$

d'où l'on déduit

$$
\left\{\begin{array}{l}
\eta_{1}=\xi_{1}-\bar{\xi}_{2} \\
\eta_{2}=\xi_{2}+\bar{\xi}_{1}
\end{array}\right.
$$

$\bar{\xi}_{1} \bar{\xi}_{2}$ désignant les quantités respectivement conjuguées de $\xi_{1}$ et $\xi_{2}$.

Il suffit donc, pour obtenir des $d s^{2}$ applicables sur des surfaces de $E_{4}$ définies par les équations (2) réelles, de prendre pour $\xi_{1}$ et $\xi_{2}$ des nombres complexes quelconques dont les parties réelles et imaginaires sont fonctions des deux variables réelles $u$, $v$, et pour $\eta_{1}, \eta_{2}$ les quantités définies par les équations (24).

Les $d s^{2}$ en question affectent ainsi la forme

$$
d s^{2}=d \xi_{1} d \eta_{2}-d \xi_{2} d \eta_{1}=d \xi_{1} d \xi_{1}+d \xi_{2} d \bar{\xi}_{2}
$$

Ainsi, par exemple, prenons

$$
\xi_{1}=e^{i \theta}, \quad \xi_{2}=e^{i \varphi} \quad(\theta, \varphi=\text { fonctions réelles de } u \text { et } v) ;
$$

on aura alors

$$
\eta_{1}=e^{i \theta}-e^{-i \varphi}, \quad \eta_{2}=e^{i \varphi}+e^{-i \theta},
$$

d'où

$$
d s^{2}=d \theta^{2}+d \varphi^{2} \quad \text { (variété de courbure nulle) }
$$

et par suite, d'après les formules (2), pour les surfaces représentatives de ce $d s^{2}$ 
PaUL VINCENSInI: Sur le plongement de certaines variétés riemaniennes, etc.

dans $E_{4}$ les équations

$$
x_{1}=\cos \varphi, \quad x_{2}=-\sin \varphi, \quad x_{3}=-\cos \theta, \quad x_{4}=\sin \theta
$$

Les formules (26) fournissent une réalisation concrète des variétes à deux dimensions de courbure nulle dans l'espace à dimensions, signalée pour la première fois W. Killing (voir E. CARTAN [12]).

Cherchons, d'une façon générale, les couples de nombres complexes $\left(\xi_{1}, \xi_{2}\right)$ pour lesquels les $d s^{2}$ de la forme (25) sont susceptibles d'être plongés dans $E_{4}$ par application des formules (2) où $\eta_{1}$ et $\eta_{2}$ ont les expressions (24).

Remarquons que, d'après les formules $(23)$ et $\left(22^{\prime}\right)$, on a

$$
\overline{O M}=\sqrt{\left|\xi_{1}\right|^{2}+\left|\xi_{2}\right|^{2}}
$$

$\left|\xi_{1}\right|^{2}$ et $\left|\xi_{2}\right|^{2}$ étant les carrés des modules des nombres complexes $\xi_{1}$ et $\xi_{2}$.

Si donc on prend $\xi_{1}$ et $\xi_{2}$ sous les formes

$$
\xi_{1}=A e^{i \theta}, \quad \xi_{2}=B e^{i \varphi},
$$

où $\theta, \varphi, A, B$ sont des fonctions réelles des variables $u, v$ auxquelles est rapporté le $d s^{2}$ envisagé, on devra donc avoir, pour que le plongement considèré ait lieu sur une hypersphère de centre $O$ (et de rayon 1 par exemple) de $E_{4}$ :

$$
A^{2}+B^{2}=1
$$

ce qui permet de poser

$$
A=\cos ^{2} \alpha, \quad B=\sin ^{2} \alpha .
$$

Il faudra done que

$$
\xi_{1}=\cos \alpha e^{i \theta}, \quad \xi_{2}=\sin \alpha e^{i p},
$$

et d'après $(24)$

$$
\begin{aligned}
& \eta_{1}=\cos \alpha e^{i \theta}-\sin \alpha e^{-i \varphi}, \\
& \eta_{2}=\sin \alpha e^{i \varphi}+\cos \alpha e^{-i \theta}
\end{aligned}
$$

Le $d s^{2}(25)$ correspondant aux expressions précédentes de $\left(\xi_{1}, \xi_{2}\right),\left(\eta_{1}, \eta_{2}\right)$ se calcule sans peine. En posant $\xi_{1}=u, \xi_{2}=v, \alpha=$ fonction differentiable queleonque de $u$ et $v$, on obtient la forme suivante:

$$
d s^{2}=d \alpha^{2}+\cos ^{2} \alpha d u^{2}+\sin ^{2} \alpha d v^{2}
$$


soit

$$
d s^{2}=\left[\left(\frac{\partial \alpha}{\partial u}\right)^{2}+\cos ^{2} \alpha\right] d u^{2}+2 \frac{\partial \alpha}{\partial u} \frac{\partial \alpha}{\partial v} d u d v+\left[\left(\frac{\partial \alpha}{\partial v}\right)^{2}+\sin ^{2} \alpha\right] d v^{2}
$$

Toutes les variétés définies par (27) admettent un plongement hypersphérique réel dans $E_{4}$, défini par les équations (2) qui s'écrivent ici:

$$
\left\{\begin{array}{l}
x_{1}=\sin \alpha \cos v, \\
x_{2}=-\sin \alpha \sin v \\
x_{3}=-\cos \alpha \cos u \\
x_{4}=\cos \alpha \sin u
\end{array}\right.
$$

Les quatre quantités $\left(x_{1}, x_{2}, x_{3}, x_{4}\right)$, étant telles que $\sum_{i=1}^{4} x_{i}^{2}=1$, peuvent être regardées comme les coordonnées de Weierstrass d'un espace elliptique rapporté au tétraèdre fondamental $\left[A_{1}(1,0,0,0), A_{2}(0,1,0,0), A_{3}(0,0,1,0), A_{4}(0,0,0,1)\right]$. On peut done interpréter les équations (28) comme définissant les applications réelles des variétés dont le $d s^{2}$ a la forme (27) dans l'espace elliptique. Et l'on voit aussitôt qu'à l'exception des conoïdes droits de ce dernier espace (dont les génératrices s'appuient sur les deux axes $A_{1} A_{2}$ et $A_{3} A_{4}$ du tétraèdre fondamental) toutes les surfaces réelles de l'espace elliptique sont données par les équations (28).

Remarque. - Si, dans (27), on regarde $\alpha$ non plus comme une fonction de $u$ et de $v$ mais comme une nouvelle variable, on a une variété à trois dimensions, dont les équations (28) définissent une représentation concrète dans l'espace elliptique à trois dimensions, représentation qui affecte la forme d'un système triple orthogonal de cet espace, dont les trois familles de surfaces sont ( $u=$ const., $v=$ const., $\alpha=$ $=$ const.), et qui n'est autre que celui que L. BIANCHI [13] a placé à l'origine d'une extension à l'espace elliptique des surfaces de Péterson de l'espace euclidin ordinaire.

Les équations des surfaces fournissant cette extension, que l'on obtient en prenant pour $\alpha$ dans les équations (28) l'expression $\alpha=\operatorname{arctg} U / V$ ( $U$ et $V=$ fonct. de $u$ et $v$ respectivement), sont

$$
\begin{array}{ll}
x_{1}=\frac{U}{\sqrt{U^{2}+V^{2}}} \cos v, & x_{2}=-\frac{U}{\sqrt{U^{2}+V^{2}}} \sin v \\
x_{3}=-\frac{V}{\sqrt{U^{2}+V^{2}}} \cos u, & x_{4}=\frac{V}{\sqrt{U^{2}+V^{2}}} \sin u .
\end{array}
$$

Pour $U$ (or $V$ ) = const., on a les surfaces de révolution de l'espace elliptique, et pour $U$ et $V$ constants la surface doublement de rotation de Clifford de ce même espace. 


\section{BIBLIOGRAPHTE}

[1] P. Vincensini, Sur une représentation quadridimensionnelle ponctuelle de l'espace règlé $\grave{a}$ trois dimensions, Annali di matematica pura ed applicata, (IV), 70 (1965), pp. 371-398.

[2] P. Vrncensinr, Rendiconti di Matematica, (4), 3, Ser. VI (1970), pp. 683-713.

[3] P. Vincensinf, Symposium G. Tzitzeica, Edit. de l'Ac. de la rép. Soc. Roumaine, Buca. rest, 1976.

[4] C. GUICHARD, Sur la déformation des surfaces, Journal de Mathématiques pures et appliquées, 1896.

[5] J. Wringarten, Aeta Mathematica, 20 (1896), pp. 159-200.

[6] G. Dä̈воUx, Surfaces, t. IV, p. 308 et suiv.

[7] E. Goursat, Annales de Toulouse, t. IV, 1891.

[8] E. Baroni, Giornale di Matematiche, t. 28, 1890.

[9] G. Gambiner, Mémorial des Sciences Mathèmatiques, Fase. XXVI.

[10] P. Vinoensini, Recherches sur la déformation des surfaces, Annales de l'Ecole Normale Sup., (3), 63, fasc. 3 (1946), pp. $255-288$.

[11] P. Vincensini, Annali di Matematica pura ed applicata, (IV), 95 (1973), pp. 321-330.

[12] E. Cartan, Leçon sur la théorie des espaces de Riemann, 2-ème éd., Paris, GauthierVillars.

[13] L. BIANchI, Congruenze di sfere di Ribaucour e superficie di Peterson, Nicolò Zanichelli, Bologna, 1928. 